\title{
Metabolism, physiology and stress defense in three aging Ins/IGF-1 mutants of the nematode Caenorhabditis elegans
}

Koen Houthoofd, ${ }^{1}$ Manuel A. Fidalgo, ${ }^{2}+$ David Hoogewijs, ${ }^{1}$ Bart P. Braeckman, ${ }^{1}$ Isabelle Lenaerts, ${ }^{1}$ Kristel Brys, ${ }^{1}$ Filip Matthijssens, ${ }^{1}$ Annemie De Vreese, ${ }^{1}$ Sylvie Van Eygen, ${ }^{1}$ Manuel J. Muñoz ${ }^{2}$ and Jacques R. Vanfleteren ${ }^{1}$

${ }^{1}$ Department of Biology, Ghent University, 9000 Ghent, Belgium ${ }^{2}$ Centro Andaluz de Biología del Desarrollo (CABD), Universidad Pablo de Olavide, Crta de Utrera Km1, 41013 Sevilla, Spain

\section{Summary}

The insulin/insulin-like growth factor-1 (Ins/IGF-1) pathway regulates the aging rate of the nematode Caenorhabditis elegans. We describe other features of the three Ins/ IGF-1 mutants daf-2, age-1 and aap-1. We show that the investigated Ins/IGF-1 mutants all have a reduced body volume, reduced reproductive capacity, increased ATP concentrations and an elevated stress resistance. We also observed that heat production is lower in these mutants, although the respiration rate was similar or higher compared with wild-type individuals, suggesting a metabolic shift in these mutants.

Key words: aging; C. elegans; Ins/IGF-1 signaling; longevity; metabolism; stress defense.

\section{Introduction}

Lifespan, development, stress resistance and metabolism of the nematode Caenorhabditis elegans are regulated by an endocrine pathway sharing homology with the insulin and insulin-like growth factor-1 (IGF-1) pathways in mammals (Kenyon et al., 1993; Lithgow et al., 1994, 1995; Riddle \& Albert, 1997; Kenyon, 2001; Pierce et al., 2001; Partridge \& Gems, 2002; Houthoofd et al., 2005). The activity of the pathway is regulated by insulinlike ligands that bind to the Ins/IGF-1 receptor DAF-2. Activation of the receptor results in autophosphorylation and subsequently the phosphorylation of the PI-3-kinase heterodimer, which is composed of the regulatory $\mathrm{p} 85$ subunit AAP- 1 and the catalytic p110 subunit AGE-1. The PIP ${ }_{2}$ and/or $\mathrm{PIP}_{3}$ formed recruit AKT1, AKT-2, SGK-1 and PDK-1 to the plasma membrane where

\section{Correspondence}

Jacques Vanfleteren, Department of Biology, Ghent University,

K.L. Ledeganckstraat 35B, 9000 Ghent, Belgium. Tel.: +32 926452 12;

fax: +32 926487 93; e-mail: Jacques.Vanfleteren@UGent.be

tDeceased.

Accepted for publication 19 January 2005
PDK-1 activates the AKT and SGK-1 kinases by phosphorylation (Alessi et al., 1997; Hertweck et al., 2004). The three kinases can antagonize the FOXO transcription factor DAF-16 by phosphorylation, but the AKT proteins preferentially regulate dauer formation whereas SGK-1 is the crucial factor for the control of development, stress response and longevity (Hertweck et al., 2004). In individuals undergoing stress, lacking food-sensing amphids or germ line cells, or carrying a mutation in one of the genes encoding proteins active in the Ins/IGF-1 pathway, DAF16 is found in the nucleus, whereas under normal growth conditions, DAF-16 resides in the cytoplasm (Henderson \& Johnson, 2001; Lin et al., 2001).

It has often been assumed that the increased metabolic activity boosted by Ins/IGF-1 signaling under conditions that favor rapid growth and reproduction would shorten life by virtue of an increased generation of reactive oxygen species (ROS) thought to cause aging (Harman, 1956). The central idea is that mitochondria are particularly susceptible to oxidative damage as they produce substantial amounts of free radicals as byproducts of normal metabolic activity. In support of this concept, several mutants of $C$. elegans with impaired mitochondrial function are long lived. A crucial question is whether the association of impaired mitochondrial function and prolonged lifespan results from fewer ROS being produced by reduced metabolic rates or from increased protection from ROS or both. Respiration rates in clk-1 mutants, a gene involved in the synthesis of ubiquinone (Ewbank et al., 1997; Jonassen et al., 2001; Rea, 2001), and in gro-1, a gene involved in the fidelity of mitochondrial translation (Lemieux et al., 2001), are not widely different from wild-type (Braeckman et al., 2002a), but mutants in isp-1, which encodes an iron-sulphur protein of complex III, are hypometabolic (Feng et al., 2001). RNAi knock-downs of nuo2 (a component of complex I), cyc-1 (a component of complex III) and cco-1 (a component of complex III) show reduced rates of respiration and ATP production. However, restoring messenger RNA to normal levels during adulthood did not elevate ATP or respiration rate, suggesting that mitochondrial function early in life establishes rates of respiration behavior and aging that persist during adulthood and that hypometabolism per se is not responsible for these effects (Dillin et al., 2002). This finding is consistent with recent studies reporting that high aerobic respiration is not necessarily linked to increasing free radical generation. Indeed, at low rates of respiration the mitochondrial inner membrane potential is very high, resulting in high ROS generation. Membrane potential drops as respiration intensifies, releasing less ROS (Korshunov et al., 1997; Brand, 2000; Nicholls, 2002). Alternatively, increased protection from ROS might 
underlie the prolonged lifespan of mutants with impaired mitochondrial function. This explanation was proposed for the isp1 mutants (Feng et al., 2001). However, Lee et al. (2003) found that RNAi inactivation of NADH cytochrome Q oxidoreductase B18 (complex II), ubiquinol-cytochrome b reductase (complex III), cytochrome c oxidase Viic (complex IV), cytochrome c oxidase Vb (complex IV), cytochrome c oxidase IV (complex IV), mitochondrial ribosomal subunit, cytochrome $c$ heme lyase and two mitochondrial carriers enhanced resistance to hydrogen peroxide but not to paraquat. Thus the mechanism by which impaired mitochondria extend lifespan appears to be more complex than simply increasing global defense against ROS.

We have previously reported that daf-2(e1370) mutants dissipated less heat per unit oxygen consumed and had elevated levels of ATP relative to wild-type worms. In addition, homogenates of daf-2(e1370) displayed higher SOD and catalase activities and they were able to reduce the dye 2,3-bis (2methoxy-4nitro-5sulfophenyl)-2H-tetrazolium-5-carboxyanilide (XTT) faster than did homogenates obtained from wild-type worms (Houthoofd et al., 2005). It is likely that reduced Ins/IGF-1 signaling causes these effects. However, other explanations cannot be excluded, e.g. these effects might be allele-specific, or they might be mediated by a distinct pathway emanating from DAF-2 and not involving PI-3-kinase. Compelling evidence has been advanced supporting the existence of such a parallel signaling pathway (Gems et al., 1998; Wolkow et al., 2002; Hertweck et al., 2004).

To investigate these possibilities we measured the effects of the aap-1(m889) and age-1(mg305) mutants in addition to daf-2(e1370) on physiology, metabolism and stress defense. $m 889$ is the only allele of aap-1 identified thus far. It likely causes a severe defect in AAP-1 because it truncates the protein after the first of two SHC boxes (Wolkow et al., 2002). daf2(e1370) and age-1(mg305) animals share a number of strong phenotypes including exceptional longevity and sensitivity to high osmolarity. The mg305 allele was also chosen as a result of an ongoing investigation (K.H. and A. Wolkow, unpublished results) of the effect of tissue-specific expression of age- 1 transgenes on the physiology, metabolism and stress defense of C. elegans.

\section{Results}

\section{Body size, reproductive capacity and lifespan}

Wild-type animals continued to grow in body length and body width for the first 3-4 days of adulthood and showed few, if any, changes in size at later ages (Fig. 1A-C). The Ins/IGF-1 mutants stopped growing 2 days earlier. The size of aap-1 animals remained essentially invariant thereafter, but daf- 2 and age-1 animals shortened substantially with increasing age. In all, the Ins/IGF-1 mutants had body volumes that were reduced by $32-42 \%$ between days 4 and 10 of adulthood. The brood size of daf-2(e1370), age-1(mg305) and aap-1(m889) animals was reduced by 72,98 and $30 \%$, respectively, independent of the growing temperature (Table 1). Average lifespan was increased by 60, 297 and 188\%, respectively (Fig. 1D).
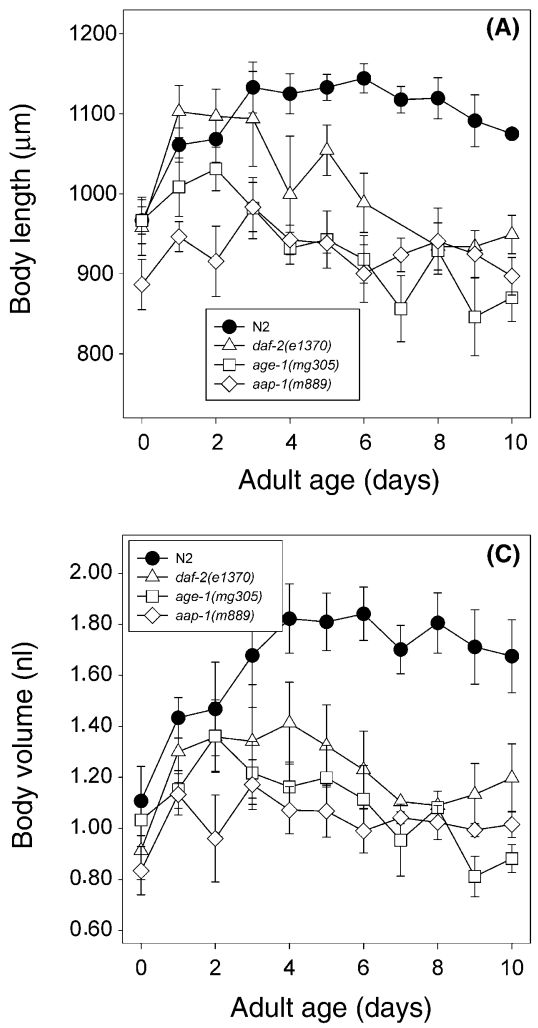
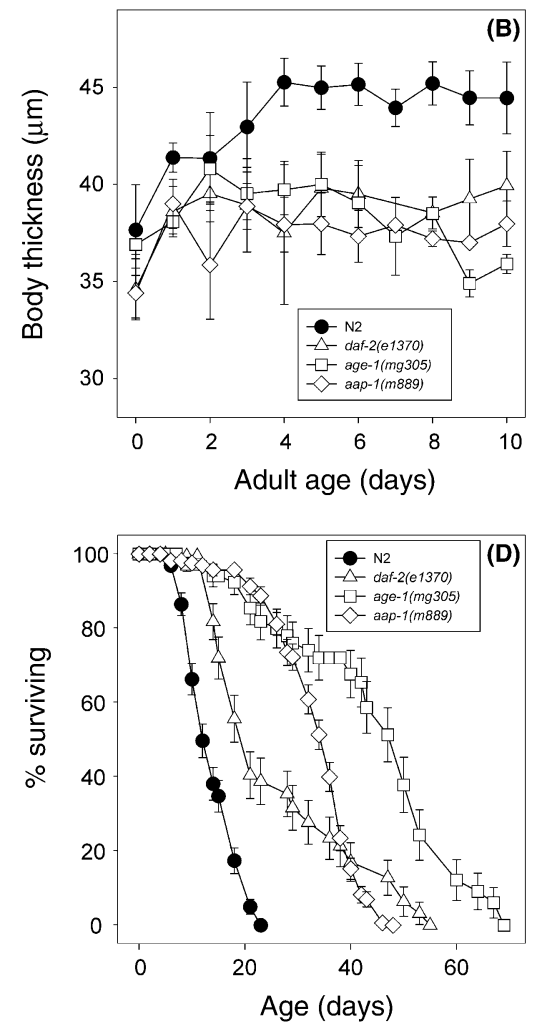

Fig. 1 Body sizes and lifespan of wild-type (N2) daf-2(e1370), age-1(mg305) and aap-1(m889) animals as a function of age. (A) Body length; (B) body width; (C) body volume; (D) survival of the four strains at $22.5^{\circ} \mathrm{C}$. Average lifespan (days) $\pm \mathrm{SE}$ (n) are: N2: $11.9 \pm 0.5(121)$, daf-2(e1370): $19.1 \pm 1.3(200)$, age- $1(\mathrm{mg} 305): 47.3 \pm 1.6(200)$, aаp-1(m889): $34.2 \pm 0.7$ (180). 
Table 1 Brood size of wild-type and Ins/IGF-1 mutants. Worms were grown at 20 or $16^{\circ} \mathrm{C}$ until L4 and subsequently shifted to 22.5 or $25^{\circ} \mathrm{C}$ to prevent dauer formation. Brood size was decreased substantially at both growing temperatures, in accordance with earlier results (Gems et al., 1998)

\begin{tabular}{lcccr}
\hline Strain & $20 \rightarrow 22.5^{\circ} \mathrm{C}$ & $\% \mathrm{~N} 2$ & $16 \rightarrow 25{ }^{\circ} \mathrm{C}$ & $\% \mathrm{~N} 2$ \\
\hline $\mathrm{N} 2$ & $241 \pm 39$ & 100 & $156 \pm 19$ & 100 \\
daf-2(e1370) & $64 \pm 21$ & 27 & $47 \pm 12$ & 30 \\
age-1(mg305) & $6 \pm 9$ & 3 & $3 \pm 9$ & 2 \\
aap-1(m889) & $170 \pm 32$ & 71 & $107 \pm 20$ & 69 \\
\hline
\end{tabular}

\section{Metabolic rate}

We examined the metabolic rate of the Ins/IGF-1 mutants by measuring respiration rate and heat production. Oxygen consumption declined steadily with age in N2 animals but much less so in daf-2(e1370) and aap-1 (mg305) animals. Consequently, the mutant worms consumed more oxygen than did wild-type from day 3 onwards (Fig. 2A; $P<0.003$ ). Unfortunately, we were not able to determine respiration of age-1 (mg305) animals because the size of the aging cohort was prohibitively small, owing to the low fertility of this strain. Heat dissipation measurements are more sensitive and could be performed for the age-1 animals as well. All three Ins/IGF-1 mutants released less heat during the first 4 days (Fig. $2 \mathrm{~B} ; P<0.02$ ), but the difference disappeared as the worms grew older. Thus, the heat production rate did not follow the respiration rate. This causes a different calorimetric/respirometric (C/R) ratio, which is a measure of catabolic efficiency (Kemp \& Guan, 1997). daf-2 and aap-1 mutants have a lower $C / R$ ratio compared with wild-type worms during most of the adult life (Fig. 2C; $P<0.05$ for the age class 2-6 days of adult age). A still more pronounced difference was reported for daf-2(e1370) previously (Houthoofd et al., 2005).

\section{ATP content}

It is generally assumed that ATP production rate and ATP consumption rate are tightly coupled, resulting in a constant ATP content. However, the results shown in Fig. 3(A) indicate that this is not the case: first, ATP concentrations steadily decline with age in wild-type worms, resulting in a $50 \%$ decrease every 4.5 days. Secondly, the three investigated mutations also influence the ATP content of worms. The mutants have increased ATP contents from day 1 of adult life onwards ( $t$-test: $P<0.05)$ and the ATP content does not show an age-dependent decrease in these strains from day 3 onwards (Fig. 3A; F-test: $P>0.1)$.

\section{XTT-reduction capacity}

Whereas XTT reduction capacity declined steadily with age in wild-type animals, no such decline was seen in any of the Ins/ IGF-1 mutants (Fig. 3B; F-test: $P>0.5$ ). This effect causes a higher reduction in capacity during most of the adult life of the
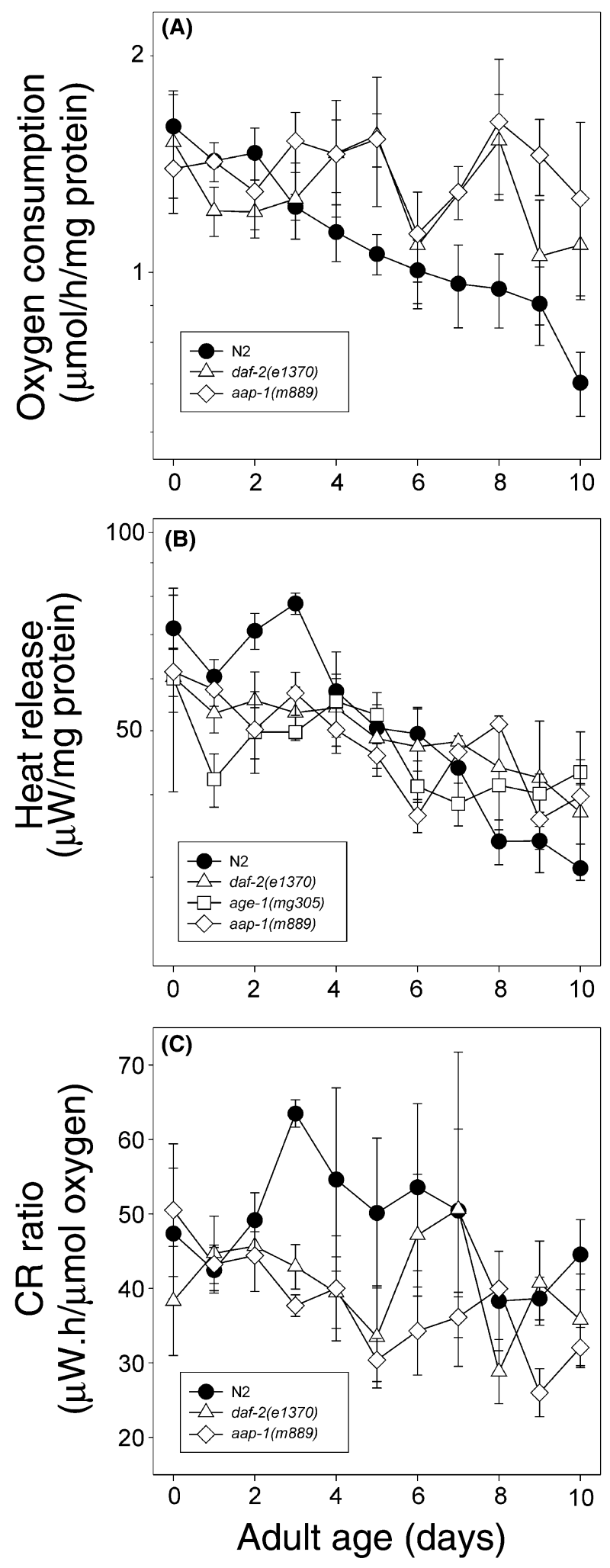

Fig. 2 Metabolic rate and C/R ratio of aging wild-type and Ins/IGF-1 mutants. (A) Respiration rate; (B) heat production; (C) C/R ratio. 

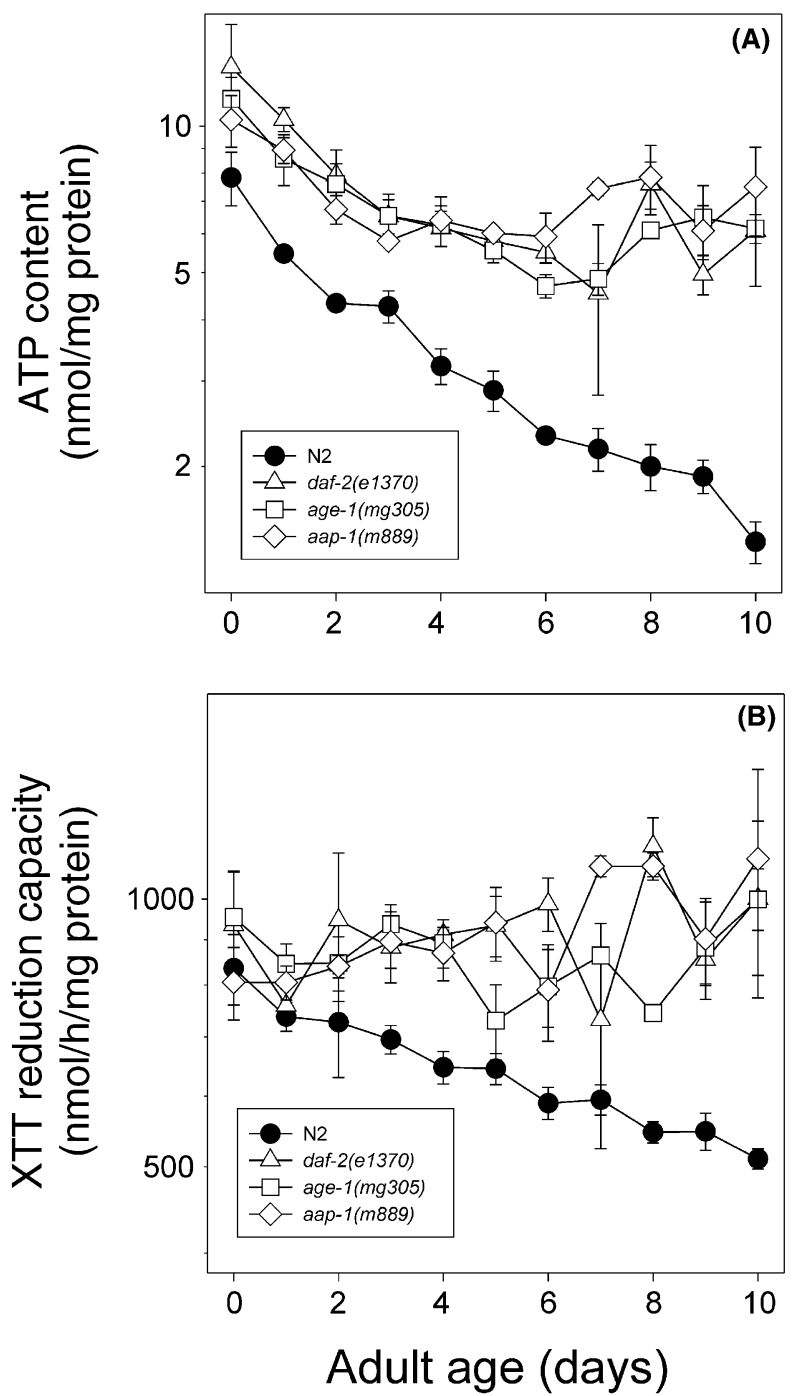

Fig. 3 (A) ATP concentration in wild-type and the three Ins/IGF-1 mutants during the adult life trajectory. (B) XTT reduction capacity in the four strains during the aging process.

Ins/IGF-1 mutants. The XTT reduction rates decreased by about $75 \%$ in all strains when $30 \mathrm{U} \mathrm{mL}^{-1}$ SOD from bovine erythrocytes (Sigma, St Louis, MO, USA) was added to the reaction mixture (data not shown), indicating that enzymatic reductase activity and superoxide accounted for approximately 25 and $75 \%$ of the XTT reduction, respectively.

\section{Stress defense}

Reduction of Ins/IGF-1 signaling enhanced the resistance to elevated temperature. Interestingly, age-1(mg305), the longest lived mutant strain under non-stressed conditions, exhibited superior resistance to elevated temperature (Fig. 4A). All three Ins/IGF-1 mutants had SOD activity levels that were about twice as high as those measured in wild-type animals (Fig. 4B; $P<$ 0.001). Catalase activity was also elevated in all three mutant strains (Fig. 4C; $P<0.001$ ).
(A)
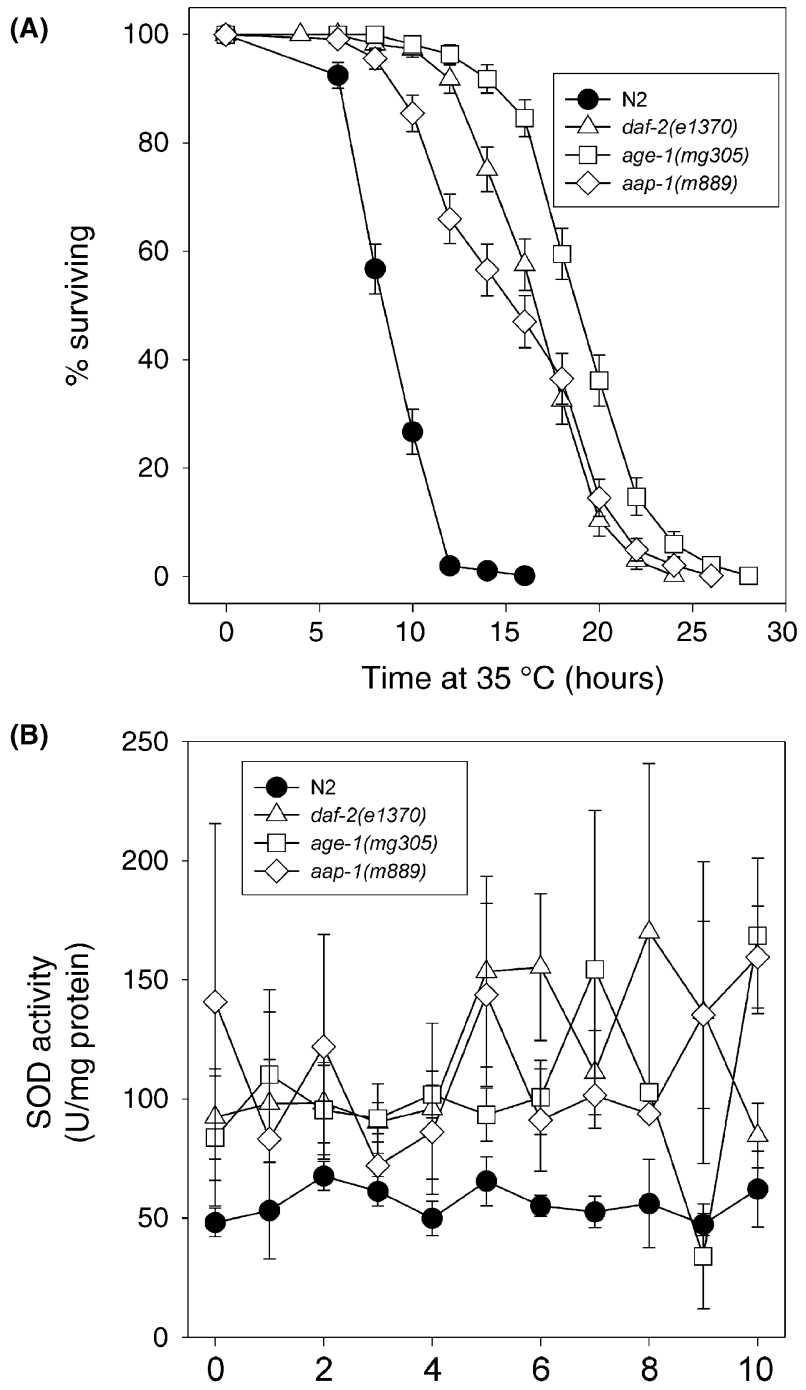

(C)

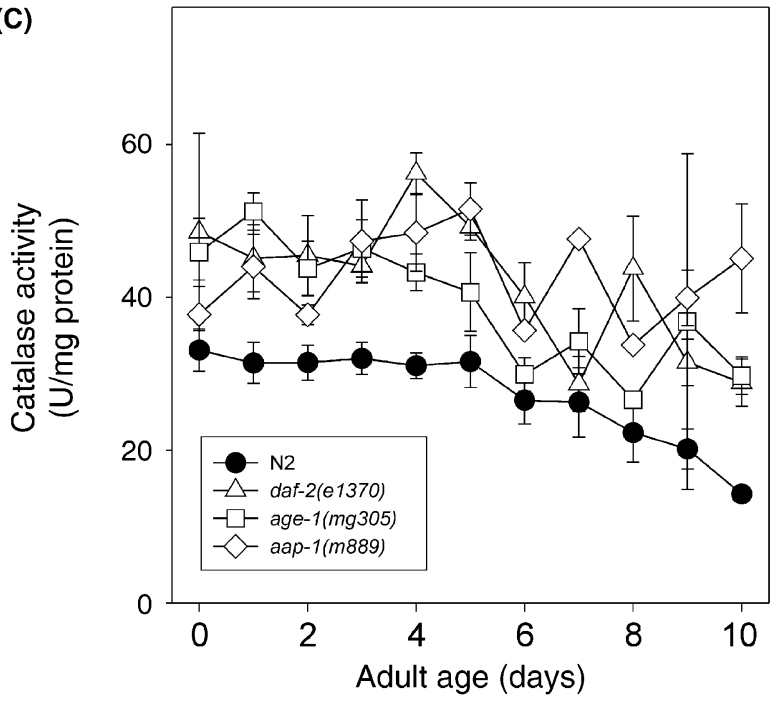

Fig. 4 (A) Resistance to elevated temperature $\left(35^{\circ} \mathrm{C}\right)$ of wild-type and Ins/lGF-1 mutants. Average survival time (hours) \pm SE $(n)$ are: N2: $8.4 \pm 0.3$ (120), daf-2(e1370): $16.6 \pm 0.4$ (120), age-1(mg305): $18.8 \pm 0.4$ (120), aap1(m889): $15.4 \pm 1.0$ (120). (B) SOD activity in aging wild-type and Ins/IGF-1 mutants. (C) Catalase activity in the four strains during the aging process. 


\section{Discussion}

We found that all three Ins/IGF-1 mutants had a reduced body size relative to wild-type animals. Van Voorhies \& Ward (1999) also found smaller body sizes for daf-2(e1370) animals, but McCulloch \& Gems (2003) reported larger body sizes for 1-dayold daf-2(e1370) adults. A number of methodological differences can be invoked to explain this discrepancy. McCulloch and Gems maintained the worms on agar with a lawn of Escherichia coli cells as a food source whereas in our study the worms were maintained in liquid culture consisting of $E$. coli in S medium.

Differences in culture temperature, however, are likely to have a greater impact. We raised the worms on agar plates at $17^{\circ} \mathrm{C}$ until they had reached the fourth larval stage and transferred them to liquid culture at $24{ }^{\circ} \mathrm{C}$, whereas McCulloch and Gems maintained the cultures invariably at $15^{\circ} \mathrm{C}$. daf-2(e1370) animals are nearly wild-type at low temperature but are dauerconstitutive and exhibit several other defects, including a severe reduction of brood size, at high temperature. The animals in the latter study therefore conceivably contained more eggs and perhaps more germ cells. On the other hand, it should be noticed that gravid aap- 1 animals had smaller body volumes than daf2 and age- 1 worms, although they contained many more eggs. It is also possible that the shift to the restrictive temperature attenuated any further increase in body size. It should also be mentioned that body volume was inferred from measurements of body volume and width, treating worms as cylinders in both studies. Body widths are very small and differences in defining the borders are bound to result in exponentially divergent estimates of volume, from calculations based on the square of the radius. Finally swelling or shrinkage artifacts associated with the measuring techniques might also contribute to the discrepancy between the McCulloch and Gems study and our measurements. Indeed, worms with mutation in the Ins/IGF-1 pathway were found to be specifically sensitive to high osmolarity (J. Wang, personal communication). The issue of body size is important because a reduced body size in all three Ins/IGF-1 mutants would indicate that Ins/IGF-1 signaling promotes body size in C. elegans, as it does in Drosophila (Böhni et al., 1999; Weinkove \& Leevers, 2000; Garofalo, 2002) and rodents (BrownBorg et al., 1996; Coschigano et al., 2000; Flurkey et al., 2001). Unfortunately, all the caveats just mentioned weaken this conclusion.

It has long been known that whole organism metabolic rate scales with a power function (b) of body mass ( $1-b$ for massspecific metabolic rate). There is still much dispute about the precise numerical value of $b$. The scaling exponent $b=2 / 3$ originally proposed by Max Rubner (1908) is consistent with the rate at which heat produced within a volume can be dissipated through the surface. Recently, White \& Seymour (2003) provided support for a scaling exponent of $b=2 / 3$ from an experimental study encompassing 619 species from 19 mammalian orders. Kleiber (1947) and Brody (1945), however, measured a greater exponent of about 3/4 in a series of mammals. Quarterpower scaling has since been based on theoretical grounds and regarded as applicable to poikilotherms and homoiotherms, single cells and even subcellular organelles as well (West et al., 1997, 1999, 2002; Gillooly et al., 2001). In the most recent attempt to resolve this argument, Demetrius (2003) postulated that energy transduction in a biological organism is constrained by processes linked to the coupling of electron transport and proton translocation, and constraints imposed by ecological and demographic forces. The mathematical outcome is that the scaling exponent will follow a $3 / 4$ rule in the case of organisms subject to scarce but dependable resources and a 2/3 rule when they are subject to ample but only temporarily available resources, i.e. both exponents are applicable, depending on the natural history of the species. In the case of $C$. elegans, the $2 / 3$ rule seems theoretically more appropriate. In the absence of compelling evidence for any numerical value of the scaling exponent, we have used the scaling exponent $b=0.72$, which was derived empirically from a series of 68 nematode species (Klekowski et al., 1972).

We have monitored respiration repeatedly in worms carrying a mutation in daf-2 or age-1 and found that the rates of oxygen consumption were either similar or higher (Vanfleteren \& De Vreese, 1996; Braeckman et al., 2002c; Houthoofd et al., 2005; this study), but never lower, in these mutants relative to the wild-type. Methodological differences may explain the contradictory results with Van Voorhies \& Ward (1999). First, Van Voorhies \& Ward (1999) ignored size differences. Second, these authors measured $\mathrm{CO}_{2}$ production, and the amounts of $\mathrm{CO}_{2}$ released per $\mathrm{O}_{2}$ consumed may be different in mutants that exhibit reduced Ins/IGF-1 signaling. SAGE analysis of dauer vs. non-dauer transcripts (Holt \& Riddle, 2003) and measurements of enzymatic activities in dauers and adult worms (O'Riordan \& Burnell, 1989) suggest that dauers actively fix $\mathrm{CO}_{2}$ in phosphoenolpyruvate, forming oxaloacetate for anabolic reactions. In addition, dauers and Ins/IGF-1 mutants have prominent fat reserves and dauers heavily rely on the combustion of stored fat that has a lower $\mathrm{CO}_{2} / \mathrm{O}_{2}$ ratio relative to carbohydrate. Because entry into the dauer stage depends on down-regulation of Ins/IGF-1 signaling, it is likely that similar metabolic shifts occur in Ins/IGF-1 mutants.

Oxygen consumption and heat production rates are equal descriptors of energy metabolism, and in principle they can be interconverted using an oxycaloric equivalent of -450 ( $\pm 5 \%$ ) $\mathrm{kJ} \mathrm{mol}^{-1} \mathrm{O}_{2}$ for all biologically useful substrates (Gnaiger \& Kemp, 1990). It should be stressed that our experimental setup precludes this approach. Respiration and heat production were measured under very divergent environmental conditions. Respiration was assayed immediately after the worms had been cleaned with sucrose and Percoll, and the contents of the measuring cell were required to be rapidly stirred for accurate measurements. In contrast, isothermal microcalorimetry required absence of agitation and at least $1 \mathrm{~h}$ of equilibration to reach a stable signal. Thus the animals were excited in one experimental environment and at rest in the other. Nevertheless, we can reasonably assume that the ratio between oxygen consumption and heat output will remain invariant among strains as long as 
the assay conditions remain unaltered. The lower $C / R$ ratio is not likely reduced by the reduced number of eggs in the gravid hermaphrodites because we observed no decrease in $C / R$ ratio in sterile $g / p-4(b n 2)$ worms (data not shown). Thus the decrease of the C/R ratio in worms in which Ins/IGF-1 signaling is reduced by mutation points to changes in the behavioral response to the assay conditions, changes in fluxes through alternative pathways of energy metabolism, or to increased efficiency of ATP generation. Rea \& Johnson (2003) hypothesized that a shift in the relative contribution of two coexisting metabolic pathways, aerobic respiration and fermentative metabolism, especially fermentative malate dismutation, might be responsible for the increased longevity seen in many animals with impaired Ins/IGF1 signaling or mitochondrial function, the tenet being that these pathways generate fewer ROS. Several lines of evidence indicate that $C$. elegans can operate to some extent anaerobically. The animals excrete lactate, acetate, succinate and propionate when incubated under anoxic conditions (Föll et al., 1990) and dauer larvae and daf-2 mutants are resistant to hypoxia in an allelespecific manner (Scott et al., 2002). Furthermore, C. elegans has a functional glyoxylate cyle (O'Riordan \& Burnell, 1990; Holt \& Riddle, 2003; Murphy et al., 2003; Wang \& Kim, 2003), and possesses the necessary tools for mitochondrial malate dismutation, including mitochondrial fumarate reductase and rodoquinone (Takamiya et al., 1999; Holt \& Riddle, 2003), cytosolic malate dismutation, and ethanol and lactate fermentation (Holt \& Riddle, 2003; Rea \& Johnson, 2003). Any anaerobic heat is predicted to increase the C/R ratio. Yet, we found that the C/ $R$ ratio was lower in the Ins/IGF mutants (Braeckman et al., 2002c; Houthoofd et al., 2005; this paper). Thus it appears that the contribution of anaerobic to total energy metabolism is likely small in the Ins/IGF-1 animals and that they possess a more efficiently operating aerobic energy metabolism.

One possible explanation for the lower $C / R$ ratios in these mutants is that they have lower proton leak rates across the mitochondrial inner membrane and thus produce ATP more efficiently. This might in turn explain the elevated ATP levels measured in these animals. Another possible reason why these mutants generate less heat is that they have reduced anabolic rates. This explanation is not tenable if it is assumed that the endothermic ATP production balances its exothermic hydrolysis. This is true for fully coupled metabolism at steady state where the rate of cellular heat dissipation then measures the rate of the catabolic processes nearly exclusively (Kemp \& Guan, 1997). However, the high ATP levels detected in the Ins/IGF-1 mutants indicate that the balance between ATP production and consumption is altered in these animals. These mutants retain higher fat stores and produce fewer offspring, suggesting that they have a reduced energetic demand for anabolic reactions, including the production of considerable quantities of yolk protein (Murphy et al., 2003). The increased fat and ATP content in germ-linedeficient mutants also supports this model (K. Houthoofd et al., unpublished results). However, the latter hypothesis cannot explain the quite similar ATP levels in the three studied Ins/ IGF-1 mutants, which have variably reduced levels of fertility.
XTT reduction capacity did not decrease in the Ins/IGF-1 mutants so that these animals had a higher XTT reduction capacity during most of their adult life. Approximately $75 \%$ of this activity is suppressible by exogenous SOD in an age- and genotypeindependent fashion. The origin of the superoxide produced in these experiments is unknown, but a respiratory source is unlikely because the homogenates were prepared in the presence of the detergent CHAPS $(0.75 \%)$. Likely sites of superoxide production under these conditions include dissociated mitochondrial complexes, small pro-oxidants and a variety of redox reactions, especially cytochrome $\mathrm{P} 450$-mediated oxidations. The balance of $25 \%$ of the XTT reduction capacity that is not suppressible by SOD is likely due to the activity of a number of diaphorases, i.e. enzymes that are capable of oxidizing $\mathrm{NAD}(\mathrm{P}) \mathrm{H}$ by a variety of artificial electron acceptors, including $\mathrm{XTT}$. Interestingly, substantial up-regulation of XTT reduction capacity was also observed for the dauer stage (Houthoofd et al., 2002a), consistent with the idea that various metabolic pathways are similarly modulated in the normal dauer stage and in mutants carrying a mutation in the Ins/IGF-1 pathway.

The elevated SOD and catalase activities that we found, including in dauers, corroborate this hypothesis. Up-regulation of catalase and SOD is frequently associated with increased longevity. Increased activities of SOD and catalase were measured in two long-lived eat-2 mutants, and in wild-type worms grown in axenic medium, which retards aging (Houthoofd et al., $2002 b, c)$ and a very large increase was found in daf-2 mutants that were grown axenically, which correlates with the 6-7-fold increase in lifespan (Houthoofd et al., 2003). Yanase et al. (2002) and Murphy et al. (2003) reported that both ctl-1 and $c t /-2$ are up-regulated in age- 1 and daf- 2 mutants. Reduction of the expression of these genes with RNAi partially suppressed the life extension imparted by mutation in daf-2 (Murphy et al., 2003). C. elegans has three genes encoding Cu/ZnSOD, sod-1, sod-4 and ZK430.3 (sod-5), and two genes encoding MnSOD, sod-2 and sod-3 (http://www.wormbase.org). The manganesecontaining enzymes likely contribute a minor fraction of total SOD activity, as little SOD activity survives cyanide poisoning (Vanfleteren, 1993). Expression analysis revealed substantial elevation of sod-3 and weak up-regulation of sod-1 in daf-2 and age-1 mutants (Honda \& Honda, 1999; Yanase et al., 2002). Thus at least one isoform each contributing to mitochondrial and cytosolic SOD activity is up-regulated in Ins/IGF-1 mutants. Recently, McElwee et al. (2003) reported the intriguing finding that ZK430.3 (sod-5) is up-regulated 12.9-fold in a daf-2 mutant and that RNAi inhibition of this gene caused a small but significant increase in the lifespan of daf-2(e1370) but has no effect on lifespan in a wild-type genetic background.

The simultaneous up-regulation of reducing and detoxifying enzyme activity as inferred from the changes in XTT, and the antioxidant SOD and catalase enzymes may indicate that reduction of Ins/IGF-1 signaling elicits the co-ordinated expression of an elaborate detoxification program, consistent with recent evidence from expression profile analysis (McElwee et al., 2004; Gems \& McElwee, 2005). 


\section{Experimental procedures}

Bristol N2 (CGC), daf-2(e1370) (CGC), age-1(mg305) (C. Wolkow) and aap-1(m889) (D. Riddle) were used in this study. C. elegans worms were grown and sampled as described in detail previously (Braeckman et al., 2002b; Houthoofd et al., $2002 a, b)$. In brief, worms were cultured at $17{ }^{\circ} \mathrm{C}$ on standard agar plates with wild-type E. coli as food source until they reached the $L 4$ stage. At that point, they were rinsed from the plates and transferred to Fernbach flasks containing $5 \times 10^{9}$ E. coli cells $\mathrm{mL}^{-1}$ and $100 \mu \mathrm{M}$ FUdR. The worm concentration was approximately $1500 \mathrm{~mL}^{-1}$ and the flasks were shaken at 120 cycles $\mathrm{min}^{-1}$ at $24^{\circ} \mathrm{C}$. The bacterial concentration was kept at a constant level by checking $\mathrm{OD}_{550}$ daily and adding $E$. coli cells from a frozen stock. A sample was taken at daily intervals for analysis from the next day (day 0) onwards. Dead worms were removed by differential centrifugation on a Percoll gradient (Fabian \& Johnson, 1994) when the fraction of dead worms exceeded $5 \%$. The sample was washed with $40 \%$ sucrose to remove bacteria. The source populations were grown on four (mutants) to six (wild-type) different occasions to account for variation caused by inadvertent environmental changes.

Lifespan analysis was performed on NGM plates with $E$. coli strain OP50. Animals were raised at $16{ }^{\circ} \mathrm{C}$ until L4 stage at which point the temperature was raised to $22.5^{\circ} \mathrm{C}$. Animals were transferred daily to fresh plates during the egg-laying period and subsequently twice per week. The fraction surviving was scored regularly. For the resistance to elevated temperature, young adults raised at $16^{\circ} \mathrm{C}$ were transferred to agar plates (without E. coli) at $35{ }^{\circ} \mathrm{C}$ and survival was scored at 2-h intervals. Survival curves for both lifespan and thermotolerance were created using the product-limit method of Kaplan \& Meier (1958). For brood size assays, ten $L 4$ animals were grown either at 20 or $16{ }^{\circ} \mathrm{C}$ and then transferred singly to the appropriate temperature. These animals were placed daily to fresh plates and the number of $\mathrm{L} 1$ progeny was counted.

Oxygen consumption rate, heat production, ATP content, XTT reduction capacity, and the activities of SOD and catalase were determined as described previously (Braeckman et al., 2002a; Houthoofd et al., 2002a,b). Oxygen consumption was measured polarographically using a six-channel respirometer from Strathkelvin (Glasgow, UK) equipped with Clark electrodes. We suspended the cleaned worms in axenic medium at 10002000 worms $\mathrm{mL}^{-1}$ and transferred $1 \mathrm{~mL}$ into each respirometer cell. Axenic medium was preferred to buffer solution because it is an appropriate food source. The worm suspensions were maintained at $24{ }^{\circ} \mathrm{C}$ and continuously stirred and oxygen concentration was monitored for 10-30 min. Oxygen-saturated water (100\% setting: $263.1 \mu \mathrm{mol} \mathrm{O}_{2} \mathrm{~mL}^{-1}$ ) and a freshly grown and dense $E$. coli culture (zero setting) were used for electrode calibration.

Metabolic heat was measured by microcalorimetry using the Thermal Activity Monitor (TAM, Biometric, Jarfalla, Sweden). A vial containing $1 \mathrm{~mL}$ of worms suspended in axenic medium and a control vial containing axenic medium only were fixed onto the measuring unit and sunk into a water bath maintained at $24^{\circ} \mathrm{C}$. Stable heat flows could be monitored for several hours after approximately $1 \mathrm{~h}$ of equilibration. Bacterial growth was suppressed by adding $250 \mathrm{U}$ penicillin and $250 \mu \mathrm{g}$ streptomycin $\mathrm{mL}^{-1}$.

ATP content was determined by monitoring the amount of light emitted when luciferin reacts with oxygen in the presence of luciferase. Flash frozen worms $(100 \mu \mathrm{L})$ were immediately submerged in boiling water for $15 \mathrm{~min}$ to destroy all ATPase activity and to allow diffusion of ATP out of the bodies. The samples were diluted with HPLC-grade water to lower the concentration of ATP and salts which interfere with the assay, and ATP was determined using the assay kit from Roche Diagnostics. The average light intensity produced between 10 and 40 min after initiation of the reaction was used to calculate the ATP content.

Worms were homogenized using a Mini-Beadbeater (Biospec Products, Bartlesvillk, OK, USA) shaken at 5000 strikes $\mathrm{min}^{-1}$ for $1 \mathrm{~min}$. The mixture was made $1 \%$ in CHAPS and clarified at 20800 r.c.f. for $10 \mathrm{~min}$ at $4{ }^{\circ} \mathrm{C}$. The capacity of the supernatant fraction to reduce the tetrazolium compound XTT to the formazan derivative was assayed in the presence of $2 \mathrm{~mm}$ each of NADH and NADPH (the reduced nicotinamide co-factors are unable to reduce XTT directly). Unidentified $\mathrm{NAD}(\mathrm{P}) \mathrm{H}$-dependent diaphorases and superoxide generated by metabolic activity in the tissue extract are XTT-reducing agents. XTT-reducing activity was therefore measured in the presence and absence of $30 \mathrm{U} \mathrm{mL} \mathrm{L}^{-1} \mathrm{SOD}$ from bovine erythrocytes to distinguish both sources of XTT-reducing activity. The assay was performed in 96-well microtiter plates and changes in absorbance were monitored at $475 \mathrm{~nm}$ for $1 \mathrm{~h}$, at $25^{\circ} \mathrm{C}$. A molar extinction coefficient of $16399.6 \mathrm{~cm}^{-1} \mathrm{M}^{-1}$ (Paull et al., 1988) was used for conversion to $\mathrm{nmol} \mathrm{h} \mathrm{mg}^{-1}$ protein.

Body volume was determined by measuring the length and thickness of 300-500 fixed worms using the RapidVue (Beckman Coulter) and using a cylindrical model for calculation. All metabolic parameters were correlated to protein content to account for differences in body mass. Respiration rate and heat production were also corrected for mass-specific differences according to the Brody-Kleiber equation (Brody, 1945; Kleiber, 1947) using a b-value of 0.72 (Klekowski et al., 1972; Braeckman et al., 2002c; see Discussion). Two-way-ANovA, Student's $t$-test and $F$-test for regression analysis were used for statistical analysis.

\section{Acknowledgments}

K.H. and B.P.B. are postdoctoral fellows with the Fund for Scientific Research-Flanders, Belgium. This work was supported by grants from Ghent University (GOA 12050101), the Fund for Scientific Research-Flanders (FWO G.002.02) and the European Commission (QLK6-CT-1999-02071). Strains in this study were kindly provided by C. Wolkow, D. Riddle and the Caenorhabditis Genetics Centre. During the preparation of this paper Manuel A. Fidalgo was tragically killed in a car accident. We will miss him forever. 


\section{References}

Alessi DR, James SR, Downes $C P$, Holmes AB, Gaffney PR, Reese $C B$, Cohen $P$ (1997) Characterization of a 3-phosphoinositide-dependent protein kinase which phosphorylates and activates protein kinase Balpha. Curr. Biol. 7, 261-269.

Bohni R, Riesgo-Escovar J, Oldham S, Brogiolo W, Stocker H, Andruss BF, Beckingham K, Hafen E (1999) Autonomous control of cell and organ size by CHICO, a Drosophila homolog of vertebrate IRS1-4. Cell 97, 865-875.

Braeckman BP, Houthoofd K, Brys K, Lenaerts I, De Vreese A, Van Eygen S, Raes H, Vanfleteren JR (2002a) No reduction of energy metabolism in Clk mutants. Mech. Ageing Dev. 23, 1447-1456.

Braeckman BP, Houthoofd K, De Vreese A, Vanfleteren JR (2002c) Assaying metabolic activity in ageing Caenorhabditis elegans. Mech. Ageing Dev. 123, 105-119.

Braeckman BP, Houthoofd K, Vanfleteren JR (2002b) Assessing metabolic activity in aging Caenorhabditis elegans: concepts and controversies. Aging Cell 1, 82-88.

Brand MD (2000) Uncoupling to survive? The role of mitochondrial inefficiency in ageing. Exp. Gerontol. 35, 811-820.

Brody S (1945) Bioenergetics and Growth. New York: Reingold.

Brown-Borg HM, Borg KE, Meliska CJ, Bartke A (1996) Dwarf mice and the ageing process. Nature 384, 33.

Coschigano KT, Clemmons D, Bellush LL, Kopchick JJ (2000) Assessment of growth parameters and life span of GHR/BP gene-disrupted mice. Endocrinology 141, 2608-2613.

Demetrius L (2003) Quantum statistics and allometric scaling of organisms. Physica A 322, 177-490.

Dillin A, Hsu AL, Arantes-Oliveira N, Lehrer-Graiwer J, Hsin H, Fraser AG, Kamath RS, Ahringer J, Kenyon C (2002) Rates of behavior and aging specified by mitochondrial function during development. Science $\mathbf{2 9 8}$ 2396-2401.

Ewbank JJ, Barnes TM, Lakowski B, Lussier M, Bussey H, Hekimi S (1997) Structural and functional conservation of the Caenorhabditis elegans timing gene Clk-1. Science 275, 980-983.

Fabian TJ, Johnson TE (1994) Production of age-synchronous mass-cultures of Caenorhabditis elegans. J. Gerontol. 49, B145B156.

Feng J, Bussiere F, Hekimi S (2001) Mitochondrial electron transport is a key determinant of life span in Caenorhabditis elegans. Dev. Cell 1, 633-644.

Flurkey K, Papaconstantinou J, Miller RA, Harrison DE (2001) Lifespan extension and delayed immune and collagen aging in mutant mice with defects in growth hormone production. Proc. Natl Acad. Sci. USA. 98, 6736-6741.

Föll RL, Pleyers A, Lewandovski GJ, Wermter C, Hegemann V, Paul RJ (1999) Anaerobiosis in the nematode Caenorhabditis elegans. Comp. Biochem. Physiol. B 124, 269-280.

Garofalo RS (2002) Genetic analysis of insulin signaling in Drosophila. Trends Endocrinol. Metab. 13, 156-162.

Gems D, McElwee JJ (2005) Broad spectrum detoxification: the major longevity assurance process regulated by insulin/IGF-1 signaling? Mech. Aging Dev. in press.

Gems D, Sutton AJ, Sundermeyer ML, Albert PS, King KV, Edgley ML, Larsen PL, Riddle DL (1998) Two pleiotropic classes of daf-2 mutation affect larval arrest, adult behavior, reproduction and longevity in Caenorhabditis elegans. Genetics 150, 129-155.

Gillooly JF, Brown JH, West GB, Savage VM, Charnov EL (2001) Effects of size and temperature on metabolic rate. Science 293, 2248-2251.

Gnaiger E, Kemp RB (1990) Anaerobic metabolism in aerobic mammaliancells - Information from the ratio of calorimetric heat-flux and respirometric oxygen flux. Biochem. Biophys. Acta 1016, 328-332.
Harman D (1956) Aging: a theory based on free radical and radiation chemistry. J. Gerontol. 11, 298-300.

Henderson ST, Johnson TE (2001) daf-16 integrates developmental and environmental inputs to mediate aging in the nematode Caenorhabditis elegans. Curr. Biol. 11, 1975-1980.

Hertweck M, Gobel C, Baumeister R (2004) C. elegans SGK-1 Is the critical component in the Akt/Pkb kinase complex to control stress response and life span. Dev. Cell 6, 577-588.

Holt SJ, Riddle DL (2003) Sage surveys C. elegans carbohydrate metabolism, evidence for an anaerobic shift in the long-lived dauer larva. Mech. Ageing Dev. 124, 779-800.

Honda Y, Honda S (1999) The daf-2 gene network for longevity regulates oxidative stress resistance and $\mathrm{Mn}$-superoxide dismutase gene expression in Caenorhabditis elegans. FASEB J. 13, 13851393.

Houthoofd K, Braeckman BP, Johnson TE, Vanfleteren JR (2003) Life extension via dietary restriction is independent of the Ins/IGF-1 signalling pathway in Caenorhabditis elegans. Exp. Gerontol. 38, 947-954.

Houthoofd K, Braeckman BP, Lenaerts I, Brys K, De Vreese A, Van Eygen S, Vanfleteren JR (2002a) Ageing is reversed, and metabolism is reset to young levels in recovering dauer larvae of C elegans. Exp. Gerontol. 37, 1015-1021.

Houthoofd K, Braeckman BP, Lenaerts I, Brys K, De Vreese A, Van Eygen S, Vanfleteren JR (2002b) No reduction of metabolic rate in food restricted Caenorhabditis elegans. Exp. Gerontol. 37, 1359-1369.

Houthoofd K, Braeckman BP, Lenaerts I, Brys K, De Vreese A, Van Eygen S, Vanfleteren JR (2002C) Axenic growth up-regulates mass-specific metabolic rate, stress resistance, and extends life span in Caenorhabditis elegans. Exp. Gerontol. 37, 1371-1378.

Houthoofd K, Braeckman BP, Lenaerts I, Brys K, Matthijssens F, De Vreese A, Van Eygen S, Vanfleteren JR (2005) Reduced insulinlike signaling and food restriction differentially affect metabolism and aging in C. elegans. Neurobiol. Aging 26, 689-696.

Jonassen T, Larsen PL, Clarke CF (2001) A dietary source of coenzyme $Q$ is essential for growth of long-lived $C$. elegans clk-1 mutants. Proc. Natl Acad. Sci. USA 98, 421-426.

Kaplan EL, Meier P (1958) Nonparametric estimation from incomplete observations. J. Am. Stat. Assoc. 53, 457-481.

Kemp RB, Guan Y (1997) Heat flux and the calorimetric-respirometric ratio as measures of catabolic flux in mammalian cells. Thermochim. Acta 300, 199-211.

Kenyon C (2001) A conserved regulatory system for aging. Cell 105, $165-168$.

Kenyon C, Chang J, Gensch E, Rudner A, Tabtiang R (1993) A C elegans mutant that lives twice as long as wild type. Nature $\mathbf{3 6 6}$, 461-464.

Kleiber M (1947) Body size and metabolic rate. Phys. Rev. 27, 511-541. Klekowski RZ, Wasilewska L, Paplinska E (1972) Oxygen consumption by soil inhabiting nematodes. Nematologica 18, 391-403.

Korshunov SS, Skulachev VP, Starkov AA (1997) High protonic potential actuates a mechanism of production of reactive oxygen species in mitochondria. FEBS Lett. 416, 15-18.

Lee SS, Lee RYN, Fraser AG, Kamath RS, Ahringer J, Ruvkun G (2003) A systematic RNAi screen identifies a critical role for mitochondria in C. elegans longevity. Nature Genet. 33, 40-48.

Lemieux J, Lakowski B, Webb A, Meng Y, Ubach A, Bussiere F, Barnes T, Hekimi $S$ (2001) Regulation of physiological rates in Caenorhabditis elegans by a tRNA-modifying enzyme in the mitochondria. Genetics 159, 147-157.

Lin K, Hsin H, Libina N, Kenyon C (2001) Regulation of the Caenorhabditis elegans longevity protein DAF-16 by insulin/IGF-1 and germline signaling. Nature Genet. 28, 139-145. 
Lithgow GJ, White TM, Hinerfeld DA, Johnson TE (1994) Thermotolerance of a long-lived mutant of Caenorhabditis elegans. J. Gerontol. 49, B270-B276.

Lithgow GJ, White TM, Melov S, Johnson TE (1995) Thermotolerance and extended life-span conferred by single-gene mutations and induced by thermal stress. Proc. Natl Acad. Sci. USA 92, 7540-7544.

McCulloch D, Gems D (2003) Body size, insulin/IGF signaling and aging in the nematode Caenorhabditis elegans Exp. Gerontol. 38, 129-136.

McElwee J, Bubb K, Thomas JH (2003) Transcriptional outputs of the Caenorhabditis elegans forkhead protein Daf-16. Aging Cell 2, 111121.

McElwee JJ, Schuster E, Blanc E, Thomas JH, Gems D (2004) Shared transcriptional signature in Caenorhabditis elegans Dauer larvae and long-lived daf-2 mutants implicates detoxification system in longevity assurance. J. Biol. Chem. 279, 44533-44543.

Murphy CT, McCarroll SA, Bargmann Cl, Fraser A, Kamath RS, Ahringer J, $\mathrm{Li} \mathrm{H}$, Kenyon C (2003) genes that act downstream of Daf-16 to influence the lifespan of Caenorhabditis elegans. Nature 424, 277-284.

Nicholls DG (2002) Mitochondrial function and dysfunction in the cell: its relevance to aging and aging-related disease. Int. J. Biochem. Cell Biol. 34, 1372-1381.

O'Riordan VB, Burnell AM (1990) Intermediary metabolism in the dauer larva of the nematode Caenorhabditis elegans 2: The glyoxylate cycle and fatty acid synthesis. Compar. Biochem. Physiol. B Biochem. Mol. Biol. 95, 125-130.

Partridge L, Gems D (2002) Mechanisms of ageing, public or private? Nature Rev. Genet. 3, 165-175.

Paull KD, Shoemaker RH, Boyd MR, Parsons JL, Risbood PA, Barbera WA, Sharma MN, Baker DC, Hand E, Scudiero DA, Monks A, Alley MC, Grote $M(1988)$ The synthesis of XTT: a new tetrazolium reagent that is bioreducible to a water-soluble formazan. J. Heterocyclic Chem. 25, 911-914

Pierce SB, Costa M, Wisotzkey R, Devadhar S, Homburger SA, Buchman AR, Ferguson KC, Heller J, Platt DM, Pasquinelli AA, Liu LX, Doberstein SK, Ruvkun G (2001) Regulation of DAF-2 receptor signaling by human insulin and ins-1, a member of the unusually large and diverse $C$ elegans insulin gene family. Genes Dev. 15, 672-686.

Rea S (2001) CLK-1/Coq7p is a DMQ mono-oxygenase and a new member of the di-iron carboxylate protein family. FEBS Lett. 509, 3894-3394.

Rea S, Johnson TE (2003) A metabolic model for life span determination in Caenorhabditis elegans. Dev. Cell 5, 197-203.
Riddle DL, Albert PS (1997) Genetic and environmental regulation of dauer larva development. In C. elegans II (Riddle DL, Blumenthal T, Meyer BJ, Priess JR, eds). Plainview, NY: Cold Spring Harbor Laboratory Press, pp. 739-768.

Rubner M (1908) Das Problem des Lebendauer und Seine Beziehungen zum Wachstum und Ernährung. Munich: Oldenbourg.

Scott BA, Avidan MS, Crowder CM (2002) Regulation of hypoxic death in C. elegans by the insulin/IGF receptor homolog DAF-2. Science 296, 2388-2391.

Takamiya S, Matsui T, Taka H, Murayama K, Matsuda M, Aoki T (1999) Free living nematodes Caenorhabditis elegans possess in their mitochondria an additional rhodoquinone, an essential component of the eukaryotic fumarate reductase system. Arch. Biochem. Biophys. 371, 284-289.

Van Voorhies WA, Ward S (1999) Genetic and environmental conditions that increase longevity in Caenorhabditis elegans decrease metabolic rate. Proc. Natl Acad. Sci. USA 96, 11399-11403.

Vanfleteren JR (1993) Oxidative stress and aging in Caenorhabditis elegans. Biochem. J. 292, 605-608.

Vanfleteren JR, De Vreese A (1996) Rate of aerobic metabolism and superoxide production rate potential in the nematode Caenorhabditis elegans. J. Exp. Zool. 274, 93-100.

Wang J, Kim SK (2003) Global analysis of dauer gene expression in Caenorhabditis elegans. Development 130, 1621-1634.

Weinkove D, Leevers SJ (2000) The genetic control of organ growth, insights from Drosophila. Curr. Opin. Genet. Dev. 10, 75-80.

West GB, Brown JH, Enquist BJ (1997) A general model for the origin of allometric scaling laws in biology. Science 276, 122-126.

West GB, Brown JH, Enquist BJ (1999) The fourth dimension of life: fractal geometry and allometric scaling of organisms. Science $\mathbf{2 8 4}$, 1677-1679.

West GB, Woodruff WH, Brown JH (2002) Allometric scaling of metabolic rate from molecules and mitochondria to cells and mammals. Proc. Natl Acad. Sci. USA 99, 2473-2478.

White CR, Seymour RS (2003) Mammal basal metabolic rate is proportional to body mass ${ }^{2 / 3}$. Proc. Natl Acad. Sci. USA 100, 4046-4049.

Wolkow A, Munoz MJ, Riddle DL, Ruvkun G (2002) Insulin receptor substrate and p55 othologous adaptor proteins function in the Caenorhabditis elegnas daf-2/insulin-like signaling pathway. J. Biol. Chem. 277, 49591-49597.

Yanase S, Yasuda K, Ishii N (2002) Adaptive responses to oxidative damage in three mutants of Caenorhabditis elegans (age-1, mev-1 and daf-16) that affect life span. Mech. Ageing Dev. 123, 1579-1587. 